\title{
Desigualdade de Renda e Eficiência Técnica na Geração de Bem-estar entre os Estados Brasileiros*
}

\author{
Emerson Marinho ${ }^{* *}$ \\ Francisco Soares ${ }^{* * *}$ \\ Mauricio Benegas ${ }^{* * * *}$
}

Sumário: 1. Introdução; 2. Metodologia; 3. Dados Amostrais; 4. Resultados; 5. Conclusão.

Palavras-chave: bem-estar; eficiência técnica; desigualdade de renda.

\section{Códigos JEL: C61; D63.}

Este artigo estima as medidas de eficiência técnica dos estados brasileiros na geração de bem-estar, entre os anos de 1986 e 1998, utilizando o método não-paramétrico Data Envelopment Analisys (DEA). São utilizadas como medidas de bem-estar a medida de Sen e, alternativamente, o PIB per capita e o Índice de Desenvolvimento Humano (IDH). Adicionalmente, são calculadas as medidas de desigualdade de renda inter/intra-regional de Theil para se analisar a trajetória da desigualdade no Brasil como uma determinante do bem-estar. Comparando as medidas de desigualdade no início e no final do período, nota-se que apenas nas regiões nordeste e sudeste ocorreram reduções significativas da desigualdade e que as medidas de desigualdade totais não sofreram qualquer modificação significativa durante o período analisado. Quanto às medidas de eficiência na geração de bem-estar, o modelo utilizando a medida de bem-estar de Sen aponta os estados de São Paulo, Rio Grande do Sul, Amazonas, Roraima e Amapá como sendo os mais eficientes na geração de bem-estar. O modelo com PIB per capita, replica basicamente os resultados anteriores. Finalmente, utilizando o IDH, os estados acima citados, mantém-se ainda como os mais eficientes com exceção do estado de São Paulo.

In this paper we utilize the Data Envelopment Analysis (DEA), a non-parametric method, to estimate measures of technical effici-

\footnotetext{
${ }^{*}$ Artigo recebido em set. 2002 e aprovado em fev. 2004.

*** Professor do Curso de Pós-Graduação em Economia - CAEN/UFC. E-mail: emarinho@ufc.br

*** Doutorando pelo Curso de Pós-Graduação em Economia - CAEN/UFC.

E-mail: fsoaresdelima@uol.com.br

${ }^{* * * * *}$ Mestre em Economia pelo Curso de Pós-Graduação em Economia - CAEN da Universidade Federal do Ceará. E-mail: benegas@bol.com.br
} 
ency of the Brazilian states in the generation of welfare between 1986 and 1998. The basic welfare measures considered include the measure developed by Sen and, alternatively, GDP per capita and the Human Development Index (HDI). Additionally, Theil's inter and intra-regional income inequality measures are calculated so that the path of income inequality as a determinant of welfare could also be analyzed for Brazil. Comparing these inequality measures in the beginning and in the end of the period in analysis, we note that only in the Northeast and in the Southeast was that inequality was significantly reduced. And, regarding the measures of efficiency in the generation of welfare, the model using Sen's measures or GDP per capita indicate that the states of São Paulo, Rio Grande do Sul, Amazonas, Roraima, and Amapá are the most efficient among all Brazilian states. When the HDI is considered, the same states are the most efficient with the exception of São Paulo.

\section{Introdução}

O debate sobre crescimento econômico e bem-estar tem se destacado nas duas últimas décadas. Até então, a teoria do crescimento econômico concentrava-se na evolução de um único parâmetro da distribuição da renda, mais especificamente, na evolução da renda média ou da renda do agente representativo. Em parte, esta escolha se deve à aceitação da hipótese de Kuznets (1955) baseada na proposição de que, se a desigualdade entre dois setores de uma economia é maior que a desigualdade intra-setorial, sua dinâmica seria determinada pela movimentação da força de trabalho de um setor para o outro. Primeiramente, a desigualdade aumentaria com o início da transferência da força de trabalho do setor menos produtivo para o mais produtivo. Posteriormente, a desigualdade diminuiria quando a maioria dos trabalhadores estivessem trabalhando no setor mais produtivo. Desta forma, a relação entre crescimento e desigualdade teria a forma de um "U" invertido.

Aceitando-se a hipótese de Kuznets, a política de desenvolvimento poderia ser resumida na promoção do crescimento econômico e, este, por fim promoveria a redução da desigualdade. Tendo-se a renda elevada e melhor distribuída, o problema da pobreza seria resolvido. Alguns trabalhos recentes apresentam evidências favoráveis à esta hipótese. Ravallion e Chen (1997) distribuíram em quatro quadrantes as observações referentes à variação no consumo médio e ao índice de pobreza. Eles verificaram que quase todas as observações localizaram-se no primeiro e no terceiro quadrantes. Desta forma, concluíram pela existência de uma correlação negativa entre crescimento e pobreza. Dollar e Aart (2000) pesquisando 
92 países nos últimos 40 anos encontraram que a taxa de crescimento da renda média dos indivíduos mais pobres foi igual a taxa de crescimento da renda média geral e, portanto, o crescimento reduziu a pobreza.

Entretanto, Deininger e Squire (1998), usando um painel de países no período entre 1960 e 1992, encontraram que não havia suporte para a hipótese de Kuznets em torno de $90 \%$ dos países investigados. Eles mostraram que em 5 países, entre eles o Brasil, a hipótese é confirmada; em 4 países, a relação entre renda e desigualdade tem o formato de "U" e em 40 países não há relação significativa entre renda e desigualdade.

Trabalhando especificamente com países em desenvolvimento, diversos estudos apresentam evidências da relação negativa entre crescimento econômico e pobreza. Lipton e Ravallion (1995) e Thorbecke e Hong-Sang (1996), constatam a importância do crescimento por setor econômico sobre a redução da pobreza. Estes dois últimos autores mostraram que o crescimento na agricultura e no setor de serviços contribuíram mais para a redução da pobreza que o crescimento do setor industrial.

A necessidade do levantamento de evidências a respeito da relação entre crescimento econômico e bem-estar origina também uma questão prática. O problema de se mensurar um fenômeno complexo como desenvolvimento econômico ou bemestar tem sido um desafio. Muitas medidas foram propostas, todas elas com um elenco de vantagens e limitações. Em muitos trabalhos, as medidas de bem-estar se baseiam em critérios de eficiência e de justiça social.

Dentre os indicadores de eficiência produtiva os mais simples e aceitos estão relacionados à renda per capita. Por sua vez, as medidas de desconcentração da renda são as medidas de justiça social mais utilizadas.

Desde o início dos anos 90, a Organização das Nações Unidas (ONU) vem divulgando o Índice de Desenvolvimento Humano (IDH) como uma medida que permita comparar o padrão de vida das nações ou regiões. Este índice se baseia em três critérios: na renda per capita, como critério de eficiência produtiva e, como critérios de justiça social, a educação (taxa de matrícula e alfabetização) e a longevidade.

No Relatório de Desenvolvimento Humano (RDH) de 2003, o Programa das Nações Unidas para o Desenvolvimento (PNUD) faz uma advertência sobre o ritmo de diminuição da pobreza no Brasil. Se o ritmo de redução da pobreza não for acelerado, não será atingida a meta de, até 2015, reduzir para 4,95\% da população o número de pessoas vivendo com renda inferior a 1 US\$/dia.

Normalmente a existência de índices elevados de pobreza está associada à baixa produtividade e, conseqüentemente, baixa renda per capita. Entretanto, no Brasil, 
como mostra Barros et alii (2001), a incidência de pobreza é maior que na maioria dos países que têm renda per capita semelhante. A desigualdade na distribuição de renda é responsável pelo fato do crescimento econômico ser relativamente incapaz na redução da pobreza, isto é, o efeito do crescimento econômico sobre a redução da pobreza é menor no Brasil que em outros países que alcançaram o mesmo nível de renda.

Nas duas últimas décadas, a produtividade dos estados brasileiros têm aumentado. Marinho e Benegas (2002), estimando o índice de produtividade total de Malmquist pelo método DEA, mostraram que, no período entre 1986 e 1998, os estados apresentaram ganhos de produtividade, aumentaram os seus estoque de insumos e, portanto, aumentaram sua capacidade de gerar renda.

A capacidade de proporcionar bem-estar à uma sociedade, de acordo com os critérios de eficiência e justiça social, seria determinada por fatores técnicos e institucionais que determinam a produção e a distribuição de riquezas. Mais precisamente, a capacidade produtiva é definida, entre outras coisas, pela tecnologia disponível e pelo nível de eficiência no emprego dos fatores de produção. Por sua vez, os determinantes da distribuição da renda são a remuneração e a distribuição dos fatores de produção entre os indivíduos. Uma sociedade que seja eficiente na produção e que, entretanto, seja ineficiente na distribuição da riqueza seria ineficiente na produção de bem-estar. O contrário também produziria ineficiência.

Isto posto, é justificável se falar em uma fronteira de produção de bem-estar. Esta fronteira seria composta pelo conjunto de pontos referentes ao nível de bemestar potencial máximo associado a uma determinada combinação de insumos. Ela seria determinada pela tecnologia de produzir e distribuir riqueza.

Usando o conceito de fronteira de produção estocástica, Marinho e Benegas (2002) mostraram que a produtividade total dos fatores no Brasil aumentou, principalmente, devida à variação tecnológica, no período entre 1986 e 1998. Além disto, mostraram também que a trajetória de produtividade é influenciada pela abertura econômica e que após 1992 a produtividade dos estados e regiões passou a crescer mais rapidamente. Assumindo o transbordamento da tecnologia no país, isto deveria implicar num aumento conjunto do nível de bem-estar de todos os estados. Entretanto, a existência de ineficiência na produção e na distribuição da renda num grupo de estados pode minimizar os ganhos de bem-estar resultantes do aumento da capacidade produtiva. 
A ineficiência acima discutida explicaria o fato do Brasil apresentar níveis de bem-estar abaixo da maioria dos países com características econômicas semelhantes a sua (Barros et alii, 1997). Esta ineficiência pode não estar perfeitamente distribuída geograficamente. Assim sendo, seria desejável conhecer onde a ineficiência está concentrada.

Neste sentido, este trabalho tem por objetivo geral estudar a trajetória da geração de bem-estar dos estados brasileiros. Mais precisamente, objetiva-se estimar a eficiência técnica dos estados brasileiros em gerar bem-estar a partir de um determinado estoque de insumos (capital e trabalho per capita) e da tecnologia produtiva disponível, no período compreendido entre 1986 e 1998. Adicionalmente, pretende-se analisar a trajetória da desigualdade na distribuição da renda média entre as unidades da federação.

O estudo da trajetória da desigualdade de renda entre estados visa verificar a possível contribuição desta variável para a eficiência na geração de bem-estar. Para esta finalidade será utilizado o índice de desigualdade de Theil. Esta medida será empregada para medir a desigualdade de renda média inter-regional e intraregional.

Para estimar as medidas de eficiência em bem-estar dos estados, será utilizada a metodologia conhecida como Data Envelopment Analisys (DEA). Esta consiste em definir uma fronteira que interliga os pontos referentes às combinações de insumos e bem-estar mais eficientes segundo o critério da produtividade.

No que se segue, além desta introdução, este artigo está organizado da seguinte forma: Na seção 2, é apresentada a metodologia utilizada, destacando-se o conceito das medidas de bem-estar, o modelo Data Envelopment Analisys que calcula as eficiências técnicas e a decomposição da medida de desigualdade da renda per capita utilizando o índice de desigualdade de Theil. Na seção 3, são descritas as variáveis usadas nas estimações e suas fontes. Na seção 4, são apresentados os resultados obtidos. Finalmente, na seção 5, são apresentadas as conclusões.

\section{Metodologia}

\subsection{Medidas de bem-estar}

Dentre as diversas medidas de bem-estar propostas na literatura, serão utilizadas três medidas neste trabalho. São elas: a medida proposta por Sen $(1973)^{1}$, o PIB per capita e o Índice de Desenvolvimento Humano (IDH).

\footnotetext{
${ }^{1}$ Veja Lambert (1993) para uma discussão detalhada sobre as propriedades da medida de bem-estar de Sen.
}

RBE Rio de Janeiro 58(4):583-608 OUT/DEZ 2004 
Tradicionalmente, a medida de bem-estar adotada é a renda per capita. Em alguns estudos mais recentes, a produtividade total dos fatores passou a substituir a renda per capita como medida de eficiência produtiva e de bem-estar. Neste tipo de análise está implícita a idéia de que a produtividade é o único determinante do bem-estar social. Neste estudo, esta abordagem será ampliada para incluir outras variáveis.

O tamanho do PIB, a população e como o PIB é distribuído entre os habitantes de um país são fatores importantes na definição do bem-estar social. Baseado nesta noção, a função de bem-estar social (FBS) de um dado país ou região com $n$ habitantes e rendas $y_{1}, y_{2}, \ldots, y_{n}$, pode ser representada por uma função $W\left(y_{1}, y_{2}, \ldots, y_{n}\right)$ que satisfaça os axiomas básicos de uma função de bem-estar. Se cada indivíduo $i$ na população tem utilidade dada por $U_{i}\left(y_{i}\right)$, então uma função de bem-estar de uma economia pode ser definida como $W=W\left[U_{1}\left(y_{1}\right), U_{2}\left(y_{2}\right), \ldots, U_{n}\left(y_{n}\right)\right]$. Se a forma funcional de $W($.) é conhecida e os dados relativos à renda de toda a população são disponíveis, seria então possível valorar o bem-estar agregado ou médio para avaliar a performance das economias.

Sob um conjunto de hipóteses e condições sobre a FBS, é possível expressá-la como uma função apenas da renda média e de um índice de desigualdade (Lambert, 1993). Desta forma, se pode definir uma função de bem estar como $W=W(\mu, I)$, onde $\mu$ representa a renda média e $I$ um índice de desigualdade de renda.

Neste artigo, a medida utilizada de desigualdade de renda entre os indivíduos será o coeficiente de Gini, G. Assim, a FBS de cada estado $j$ será definida por uma função $W_{j}=W\left(\mu_{j}, G_{j}\right)$.

Segundo Mukhopadhaya (2001), a maioria das FBS encontradas na literatura são paretianas, isto é, um aumento na renda de qualquer indivíduo aumenta o bem-estar social, desde que nenhum outro indivíduo tenha diminuição de renda. A FBS proposta por Sen possui estas propriedades. Assim sendo, no modelo 1 utilizar-se-á a medida de Sen definida como

$$
W(\mu, G)=\mu(1-G)
$$

onde $(1-G)$ é a medida de igualdade de distribuição da renda. Esta especificação particular tem sido em geral usada na literatura. Sen (1973) apresenta a intuição para esta escolha particular de função. Kakwani (1980) também providencia um sistema de axiomas sob os quais uma FBS pode ser definida como $\mu(1-G)$. Para esta função, as elasticidade de $W(\mu, G)$ com respeito a $\mu$ e $G$, são, respectivamente, 1 e $-G /(1-G)$. Deste resultado, conclui-se que esta medida é mais sensível à renda quanto menor que 0,5 for o coeficiente de Gini. Assim sendo, espera-se que esta medida de igualdade contribua significativamente para explicar a eficiência em 
gerar bem-estar dos estados brasileiros, dado que a concentração de renda nesses estados, medida por aquele coeficiente, é maior que 0,5.

Ainda que as medidas de bem-estar no modelo 1 sejam mais sensíveis às medidas de desigualdade, propõe-se, alternativamente, a utilização da renda média como uma outra medida de bem-estar. Desta forma, a análise da eficiência em bem-estar será idêntica à análise da produtividade. Esta medida será utilizada no modelo 2.

Deve-se observar que ambas medidas consideram apenas o bem-estar em função da renda auferida pelos indivíduos e desconsideram os possíveis efeitos de políticas públicas sobre o bem-estar social. Neste sentido, o modelo 3 utiliza como output o Índice de Desenvolvimento Humano. Esta medida é mais complexa do que as duas anteriores, sendo defendida como um indicador mais eficiente das condições sócio-econômicas por que, além de considerar o nível de renda, também incorpora indicadores referentes à educação (taxa de matrícula e alfabetização) e a longevidade dos indivíduos.

Na subseção 4.2, as estimativas com os três outputs serão comparadas para verificar se produzem trajetórias de eficiência semelhantes.

\subsection{Data envelopment analisys}

Para analisar o uso eficiente de algum recurso se faz necessária a definição de uma referência de eficiência técnica. Neste estudo, a medida de eficiência será obtida pela utilização do método Data Envelopment Analisys (DEA) para definir uma fronteira formada pela conexão das melhores tecnologias na utilização dos insumos para geração de bem-estar. Nesta estrutura as unidades da federação usam inputs para gerarem outputs. Os inputs são os estoques de capital e trabalho utilizados na produção e os outputs são indicadores de bem-estar.

Antes de se discutir mais detalhadamente o DEA, é necessário apresentar os conceitos de conjunto de possibilidades de produção e função distância que caracterizam o processo tecnológico de produção.

O conjunto de possibilidades de produção representa o conjunto de todos os vetores de produtos, $y \in R_{+}^{M}$, que possam ser produzidos usando o vetor dos insumos, $x \in R_{+}^{N}$. Isto é,

$$
P(x)=\{y: x \text { pode produzir } y\}
$$

é o conjunto de todas as combinações de insumos e produtos factíveis. A figura 1 ilustra o conceito de um conjunto de possibilidades de produção. Este conjunto consiste de todos os pontos entre a fronteira de possibilidades de produção (FPP) 
e o eixo dos y's. Os pontos ao longo da fronteira de produção definem um subconjunto eficiente deste conjunto. Assim sendo, os pontos $B$ e $C$ são exemplos de produção eficiente enquanto o ponto $A$ é um exemplo de ineficiência produtiva.

Para a finalidade deste estudo, o conjunto de possibilidades de produção representará o conjunto de todos os vetores de bem-estar que possam ser produzidos usando o vetor de insumos. Assume-se a existência de uma tecnologia na geração de bem-estar.

A função distância é a recíproca da medida de eficiência técnica proposta por Farrell (1957). Esta medida pode ser calculada orientada pelo insumo ou orientada pelo produto. A função distância orientada pelo produto, $D_{0}(x, y)$, é uma medida de quanto o nível de produto observado está distante do produto máximo potencial para um dado conjunto de insumos utilizados na sua produção. Formalmente, pode ser definida em função do conjunto de possibilidades de produção, $P(x)$, como:

$$
D_{0}(x, y)=\operatorname{Inf}\{\delta:(x, y / \delta) \in P(x)\}
$$

Em termos do conjunto de possibilidades de produção, a função distância orientada pelo produto é a máxima expansão do vetor de produto observado, necessária para torná-lo igual ao vetor de produto potencial máximo. ${ }^{2}$

De acordo com o diagrama na figura 1, a função distância no ponto A é definida com sendo igual a $\delta=O A / O B$ que é menor que um. Neste caso, a unidade de produção está operando ineficientemente, pois com o insumo $x$ ela poderia operar no ponto B sobre a fronteira de possibilidades de produção. Se a unidade de produção estivesse operando no ponto C, seria eficiente e a sua função distância seria igual a um.

O modelo DEA foi originalmente proposto por Charnes et alii (1978) e através dele são estimadas as medidas de eficiência técnica orientadas pelo insumo ou pelo produto para cada unidade de decisão. Neste artigo, as medidas de eficiência técnicas serão orientadas pelo produto por entender que o objetivo da sociedade é maximizar o bem-estar a partir dos recursos produtivos utilizados.

\footnotetext{
${ }^{2} \mathrm{~A}$ função distância orientada pelo insumo é definida como $D_{i}(x, y)=\sup \delta:(x / \delta, y) \in P(x)$, ou seja, é a máxima contração proporcional dos insumos necessária para que se produza o mesmo nível de produto.
} 
Figura 1

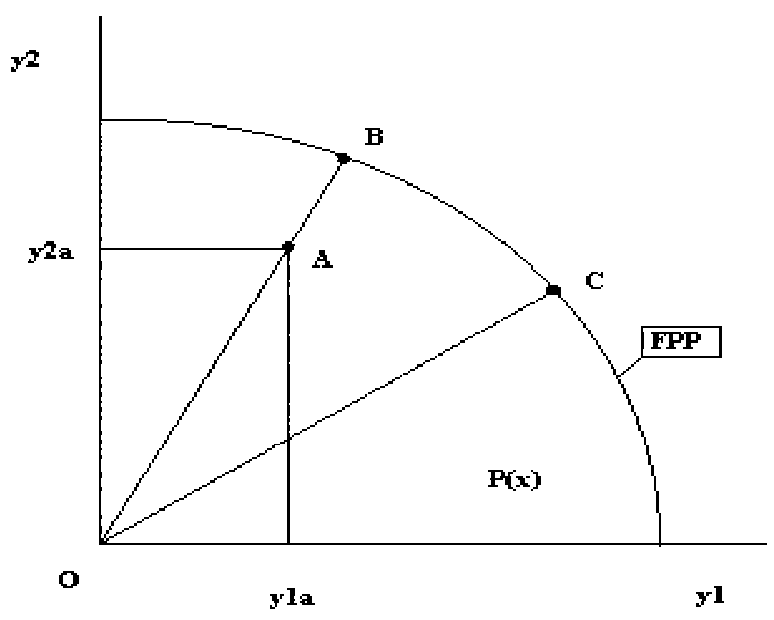

Este modelo consiste no uso de métodos de programação linear para a construção das fronteiras não paramétricas sobre os dados. As funções distâncias são então calculadas relativamente a esta fronteira, segundo Lovell (1993).

Assim sendo, denote por $y_{i}$ e $x_{i}$ os vetores de ordem (Mx1) e (Nx1) representando, respectivamente, os produtos e os insumos utilizados pela i-ésima unidade de decisão (UD). Então para esta UD pode-se obter a razão entre todos os outputs e todos os inputs, tal como $u^{\prime} y_{i} / v^{\prime} x_{i}$, onde $u$ é um vetor ( $\mathrm{M} \times 1$ ) de pesos dos outputs e $v$ é um vetor $(\mathrm{N} \times 1)$ de pesos dos inputs. Os vetores $u$ e $v$ podem ser obtidos adequadamente para que esta razão seja uma medida da produtividade total dos fatores da i-ésima UD. Os pesos ótimos são obtidos resolvendo-se o seguinte problema de programação matemática:

$$
\operatorname{Max}_{u, v}\left(u^{\prime} y_{i} / v^{\prime} x_{i}\right)
$$

sujeito à

$$
\begin{gathered}
\left(u^{\prime} y_{j} / v^{\prime} x_{j}\right) \leq 1, \quad j=1,2, \ldots, N \\
u, v \geq 0
\end{gathered}
$$

O programa acima tem por objetivo encontrar os valores de $u$ e $v$, tal que a medida de eficiência para a $i$-ésima UD seja maximizada, sujeito à restrição de 
que todas as medidas de eficiência técnica sejam menores ou iguais a um. Um problema com a particular forma em (3) é que o programa tem infinitas soluções. ${ }^{3}$ Para se evitar isto, impõe-se a restrição $v^{\prime} x_{i}=1$, e, desta forma, (3) pode ser rescrito como

$$
\operatorname{Max}_{\mu, \nu}\left\{\mu^{\prime} y_{i}\right\}
$$

sujeito à

$$
\begin{gathered}
\nu^{\prime} x_{i}=1 \\
\mu^{\prime} y_{j}-\nu^{\prime} x_{j} \leq 0, \quad j=1,2, \ldots, N \\
\mu, \nu \geq 0
\end{gathered}
$$

onde a mudança de notação de $u$ e $v$ para $\mu$ e $\nu$ é usado para evidenciar que este é um programa linear diferente. A forma em (4) é conhecida como a forma multiplicativa do modelo DEA.

Utilizando o conceito de dualidade em programação linear, pode-se derivar uma forma equivalente deste problema, ou seja, o problema (4) pode ser definido como

$$
\operatorname{Min} \theta^{k}
$$

sujeito à

$$
\begin{gathered}
y_{m}^{k} \leq \sum_{k=1}^{K} \lambda^{k} y_{m}^{k} \\
m=1, \ldots, M \\
\sum_{k=1}^{K} \lambda^{k} x_{n}^{k} \leq \theta^{k} x_{n}^{k} \\
n=1, \ldots, N
\end{gathered}
$$

${ }^{3} \operatorname{Se}\left(u^{*}, v^{*}\right)$ é uma solução então $\left(\alpha u^{*}, \alpha v^{*}\right)$ é uma outra solução. 
No modelo acima, a fronteira (ou referência) tecnológica do output para a região $k$ é construída dos dados na forma:

$T=\left\{\left(x^{k}, y^{k}\right) ; \sum_{k=1}^{K} \lambda^{k} x_{n}^{k} \leq x_{n}^{k} n=1, \ldots, N ; y_{m}^{k} \leq \sum_{k=1}^{K} \lambda^{k} y_{m}^{k} \quad m=1, \ldots, M ; \lambda^{k} \geq 0\right\}$

A tecnologia acima exibe retornos constantes de escala e é definida por um cone poliedral (fechado pela igualdade) formado pela interseção dos hiperplanos gerados pelas restrições de disponibilidade dos inputs, $\sum_{k=1}^{K} \lambda^{k} x_{n}^{k} \leq x_{n}^{k}, n=1, \ldots, N$, e pela restrição de capacidade definida por $y_{m}^{k} \leq \sum_{k=1}^{K} \lambda^{k} y_{m}^{k}, m=1, \ldots, M$. Os $\lambda^{k}$ 's são variáveis de intensidade indicando em que nível de intensidade uma atividade em particular pode ser empregada na produção de bem-estar. As restrições quanto as disponibilidades, permitem a construção de uma fronteira não paramétrica onde os pontos à esquerda e abaixo de uma isoquanta não paramétrica não são factíveis, seguindo a sugestão de Farrell (1957). A estimação da eficiência técnica orientada pelo insumo para uma determinada UD é feita resolvendo-se o problema (5). Usando a definição de função distância orientada pelo insumo e sua reciprocidade como a medida de eficiência técnica orientada pelo insumo, além da construção de $T$ em (6), pode-se rescrever o programa (5) acima como

$$
\left[D_{i}\left(x^{k}, y^{k}\right)\right]^{-1}=\operatorname{Min} \theta^{k}
$$

sujeito à

$$
\begin{gathered}
y_{m}^{k} \leq \sum_{k=1}^{K} \lambda^{k} y_{m}^{k} \\
m=1, \ldots, M \\
\sum_{k=1}^{K} \lambda^{k} x_{n}^{k} \leq \theta^{k} x_{n}^{k} \\
n=1, \ldots, N \\
\lambda^{k} \geq 0
\end{gathered}
$$


A solução do problema acima para as $K$ unidades de decisão (UD) para uma determinada amostra resulta nos resultados de eficiência técnica para cada uma delas. A fronteira é então construída a partir das UD mais eficientes e as demais posicionadas relativamente a tal fronteira. O programa acima é resolvido para cada uma das $K$ unidades de decisão, e desta forma a fronteira tecnológica nacional de bem-estar é construída.

\subsection{Decomposição da medida de desigualdade da renda per capita}

A desigualdade de produtividade entre os estados pode ser entendida como uma evidência de um desequilíbrio na absorção de tecnologia (capacidade produtiva) ou na eficiência técnica produtiva entre os estados. Alguns estados podem estar mais aptos em absorver novos processos de produção e conseguir proporcionar mais riqueza para as suas populações.

Tomando a renda per capita como uma proxy da produtividade dos estados, a análise da distribuição da renda proporciona informação sobre a desigualdade em absorção tecnológica e eficiência produtiva e, conseqüentemente, sobre um dos componentes de indicadores de bem-estar.

A desigualdade na distribuição de renda pode ser verificada sob dois pontos de vista distintos. A desigualdade pode ocorrer devido a dispersão na renda média entre os estados de uma região e/ou entre as regiões. A desigualdade total será determinada pela soma das desigualdades ocorridas em ambos os níveis.

Desta forma, será calculada uma medida de desigualdade de distribuição da renda entre as regiões e entre os estados de uma mesma região. Finalmente, será construída uma medida nacional de desigualdade composta pelas duas primeiras medidas.

A medida de desigualdade utilizada será o índice de Theil $(1989,1996)$. A escolha desta medida deve-se a propriedade da aditividade que permite que uma medida de desigualdade total seja formada pela soma das medidas de desigualdade inter e intra-grupos.

Para construir esta medida, inicialmente, denote por $Y_{i j}$ e $P_{i j}$ a renda per capita e a população do estado $i$ da região $j$. Denote também por $Y_{j}$ e $P_{j}$, respectivamente, a renda per capita e a população da região $j$. Então, $P_{j}=\sum_{i=1}^{n_{j}} P_{i j}$ e $Y_{j}=\frac{\sum_{i=1}^{n_{j}} Y_{i j} P_{i j}}{P_{j}}$, onde $n_{j}$ é o número de estados da região $j$.

Para cada região $j$, denote por $\pi_{i j}$ e $\eta_{i j}$, respectivamente, a participação da população e da renda do estado $i$ na população e na renda da região $j$. 
A medida de Theil de desigualdade na distribuição da renda entre estados de uma mesma região, $L_{j}$, é então definida como

$$
L_{j}=\sum_{i=1}^{n j} \pi_{i j} \ln \left(\frac{\pi_{i j}}{\eta_{i j}}\right)
$$

A contribuição da desigualdade dentro das regiões para a desigualdade total, aqui denominada de $L_{W}$, pode ser calculada como a média ponderada das medidas de desigualdade dentro de cada região. Assim tem-se que

$$
L_{W}=\sum_{j=1}^{K} L_{j} \frac{P_{j}}{P}
$$

onde $P$ é a população do país.

Para medir a desigualdade entre as regiões, utiliza-se uma medida semelhante a (8), na forma

$$
L_{B}=\sum_{i=1}^{n j} \pi_{j} \ln \left(\frac{\pi_{j}}{\eta_{j}}\right)
$$

em que $\pi_{j}$ e $\eta_{j}$ denotam, respectivamente, a participação da renda e da população da região $j$ na renda e população nacional.

Nestes termos, a desigualdade total é medida simplesmente pela soma de (9) e $(10)$.

\section{Dados Amostrais}

Para calcular as medidas de desigualdade (8), (9) e (10) de Theil entre os estados de uma mesma região e entre as regiões, foram utilizadas a séries de PIB per capita e a população dos estados brasileiros obtidas a partir das Contas Regionais publicadas pelo IBGE (2000a) para o período de 1986 a 1998. Não foi possível estender esta análise para os anos anteriores a 1986 em virtude destes dados só serem divulgados de cinco em cinco anos.

Para a estimação das medidas de eficiência na geração de bem-estar dos estados brasileiros, foram utilizadas como outputs as séries de PIB per capita, o complementar do Índice de Gini (Benevides e Ataliba, 2002) vezes o PIB per capita e o IDH. Este último foi obtido no site do Programa das Nações Unidas para o Desenvolvimento (PNUD) somente para os anos 1991, 1995, 1996 e 1998 . Os inputs considerados foram o consumo de energia elétrica total menos o consumo 
de energia elétrica residencial como proxy do estoque de capital, cujos dados foram extraídos dos Anuários Estatísticos do Brasil dos anos de 1987 a 1999, publicados pelo IBGE (2000). O outro input foi a série das horas trabalhadas per capita , como proxy do fator trabalho, construídas a partir dos dados da Pesquisa Nacional por Amostragem Domiciliar (PNAD), publicados pelo IBGE (2000b).

Deve-se ressaltar que esse último input pode não captar o diferencial de produtividade entre trabalhadores de regiões distintas devido, por exemplo, a possíveis diferenciais de capital humano. Neste sentido, os resultados e análise apresentados na seção seguinte poderiam ser outros caso se considerasse esse efeito.

\section{Resultados}

Nesta seção, são apresentados os principais resultados referentes ao cálculo das medidas de desigualdade de renda entre os estados e da estimação da fronteira de produção não-paramétrica de bem-estar.

\subsection{Trajetória da desigualdade na distribuição de renda per capita entre os estados e regiões}

A análise da trajetória da desigualdade no Brasil inicia-se pelo cálculo das medidas de desigualdade intra-regional através da expressão (8). Estas medidas encontram-se resumidas na tabela 1 .

De acordo com os dados da tabela 1, observa-se que a renda, na maioria dos anos da amostra, é menos concentrada na região Centro-Oeste embora, aparentemente, seja a única a apresentar uma ligeira tendência crescente de desigualdade. As regiões Centro-Oeste, Sul e Sudeste, nesta ordem, apresentam médias de desigualdade menores que a média brasileira. Ao contrário das demais regiões, Norte e Nordeste apresentam em todo o período as maiores medidas de desigualdade de renda, mas com uma tendência decrescente. Em resumo, exceto na região CentroOeste, todas as demais apresentam suaves tendências decrescentes na desigualdade de renda. 
Tabela 1

Medidas de desigualdade na distribuição de renda entre os estados por região no período de 1986 e 1998

\begin{tabular}{ccccccc}
\hline \multicolumn{7}{c}{ Medidas de Desigualdade de Theil } \\
\hline Região & Norte & Nordeste & Sudeste & Sul & Centro-Oeste & $L_{W}$ \\
\hline 1986 & 0,032026 & 0,027521 & 0,015888 & 0,003038 & 0,002434 & 0,019548 \\
1987 & 0,032684 & 0,027001 & 0,018322 & 0,002366 & 0,002043 & 0,020003 \\
1988 & 0,037092 & 0,025659 & 0,019277 & 0,002837 & 0,001837 & 0,020162 \\
1989 & 0,022712 & 0,023141 & 0,018882 & 0,002454 & 0,002487 & 0,019020 \\
1990 & 0,023540 & 0,018136 & 0,018117 & 0,001397 & 0,001941 & 0,016589 \\
1991 & 0,020434 & 0,018199 & 0,014026 & 0,001765 & 0,001420 & 0,014209 \\
1992 & 0,024853 & 0,018770 & 0,013932 & 0,003970 & 0,003499 & 0,017024 \\
1993 & 0,019254 & 0,018031 & 0,013573 & 0,005043 & 0,003832 & 0,016799 \\
1994 & 0,019245 & 0,014993 & 0,010148 & 0,004116 & 0,001774 & 0,012257 \\
1995 & 0,024410 & 0,015380 & 0,011887 & 0,002976 & 0,002621 & 0,014113 \\
1996 & 0,030107 & 0,015493 & 0,010121 & 0,001926 & 0,003102 & 0,014084 \\
1997 & 0,028574 & 0,013962 & 0,010869 & 0,001762 & 0,002709 & 0,013452 \\
1998 & 0,026154 & 0,015761 & 0,011438 & 0,001023 & 0,003544 & 0,014774 \\
Média & 0,026237 & 0,019388 & 0,014345 & 0,002667 & 0,002557 & 0,016310 \\
\hline Obs.: A coluna $L_{W}$ apresenta as medidas correspondentes à expressão (9) - \\
as medidas de contribuição da desigualdade intra-regional para a desigualdade total. \\
Fonte: Resultados calculados pelos autores.
\end{tabular}

Tabela 2

Teste de hipótese para diferença entre as médias da medida de desigualdade nos primeiros e últimos cinco anos da amostra

\begin{tabular}{lccccc}
\hline & \multicolumn{4}{c}{ Medidas de Desigualdade de Theil } \\
\hline \multicolumn{1}{c}{ Região } & Norte & Nordeste & Sudeste & Sul & Centro-Oeste \\
\hline M1 & 0,0296 & 0,0242 & 0,0180 & 0,0024 & 0,0315 \\
M2 & 0,0256 & 0,0151 & 0,0108 & 0,0023 & 0,0375 \\
M2 - M1 & $-0,0039$ & $-0,0097$ & $-0,0072$ & $-5,8 \mathrm{E}-05$ & 0,0060 \\
Desvio Padrão & 0,0034 & 0,0017 & 0,0007 & 0,0006 & 0,0043 \\
Estatística $t$ & $-1,1628$ & $-5,7079$ & $-10,7331$ & $-0,0998$ & 1,3975 \\
\hline Obs.: O valor crítico da distribuição $t$, com oito graus de liberdade, \\
associado ao nível de significância de $5 \%$ em um teste bi-caudal \\
é igual a 2,306. \\
Fonte: Resultados calculados pelos autores.
\end{tabular}

Para verificar se houve variação estatisticamente significativa na dispersão da renda nas regiões, testou-se a hipótese de que a diferença entre as médias da medida de desigualdade nos primeiros (M1) e últimos cinco (M2) anos da amostra é igual a zero. O resultado da estatística $t$ na tabela 2 mostra que a hipótese nula é aceita somente nas regiões Norte, Sul e Centro-Oeste. Entretanto, nas regiões Nordeste e Sudeste as reduções nas medidas de desigualdade foram estatisticamente significantes no nível de 5\%. Portanto, as evidências estatísticas mostram que houve redução da desigualdade de renda apenas nestas duas regiões nestes 
últimos anos. Deve-se observar que a redução da dispersão ocorreu justamente na região mais rica e na mais pobre do país. Isto poderia ser resultado de um processo de formação de dois grupos homogêneos de baixa e alta produtividade e renda.

Se a desigualdade intra-regional diminui e a desigualdade inter-regional aumenta, pode-se verificar uma deterioração da desigualdade total no país. Para esclarecer este ponto, será analisada a trajetória da desigualdade entre as regiões utilizando-se a expressão (10). Os resultados são apresentados na tabela 3.

Tabela 3

Medidas de desigualdade na distribuição de renda entre as regiões brasileiras

\begin{tabular}{cc}
\hline Ano & $L$ de Theil \\
\hline 1986 & 0,0883 \\
1987 & 0,1041 \\
1988 & 0,1094 \\
1989 & 0,1172 \\
1990 & 0,1041 \\
1991 & 0,0974 \\
1992 & 0,1059 \\
1993 & 0,0998 \\
1994 & 0,1001 \\
1995 & 0,1045 \\
1996 & 0,0935 \\
1997 & 0,1010 \\
1998 & 0,1004 \\
Média & 0,1020 \\
\hline Fonte: Resultados calculados pelos autores.
\end{tabular}

Testando a hipótese nula que a diferença entre as médias dos primeiros e últimos cinco anos é igual a zero, obtém-se uma estatística $t$ igual a $-2,086$. Então, aceita-se que houve redução na desigualdade da renda entre as regiões e rejeita-se a hipótese nula no nível de significância de $5 \%$.

Em relação às medidas de desigualdade total para o Brasil, estas são calculadas como a soma dos resultados das expressões (9) e (10) e se encontram dispostas na tabela 4 . 
Tabela 4

Medidas de desigualdade na distribuição de renda no Brasil

\begin{tabular}{cc}
\hline Ano & $L$ de Theil \\
\hline 1986 & 0,10785 \\
1987 & 0,12407 \\
1988 & 0,12961 \\
1989 & 0,13625 \\
1990 & 0,12073 \\
1991 & 0,11161 \\
1992 & 0,12295 \\
1993 & 0,11661 \\
1994 & 0,11232 \\
1995 & 0,11860 \\
1996 & 0,10760 \\
1997 & 0,11447 \\
1998 & 0,11522 \\
Média & 0,11830 \\
\hline Fonte: Resultados calculados pelos autores.
\end{tabular}

Realizando o mesmo teste de hipótese para as medidas de desigualdade total, encontra-se uma estatística $t$ igual a $-4,417$. Portanto, no nível de 5\%, conclui-se que houve redução significativa da desigualdade de renda entre os estados.

Os resultados apresentados acima mostram que houve uma redução significativa da desigualdade total da renda entre os estados. Entretanto, apenas em duas regiões a dispersão da renda entre seus estados diminuiu.

Quanto a presença ou não de efeitos regionais, pode-se demonstrar que a redução na desigualdade se deve basicamente a redução na dispersão entre regiões. Na verdade, aproximadamente, $86 \%$ da redução da desigualdade em produtividade entre os estados se deve a redução da desigualdade entre as regiões.

Esta redução na dispersão da renda pode ser entendida como uma aproximação do nível de produtividade entre as economias estaduais. Entretanto, não se pode afirmar que o bem-estar também esteja evoluindo no mesmo sentido.

\subsection{Eficiência na geração de bem-estar}

Esta seção se destina a analisar os resultados de eficiência técnica na geração de bem-estar dos estados brasileiros, levando-se em consideração as três medidas de bem-estar apresentadas na subseção 2.1.

\subsection{Modelo 1 - Eficiência usando a medida de Sen como output}

A tabela 5 apresenta os valores estimados das medidas de eficiência na geração de bem-estar dos estados brasileiros. Para facilitar a análise destas trajetórias, 
são apresentados somente os valores correspondentes às médias para os seguintes quatro períodos: 1986-89, 1990-92, 1993-95 e 1996-98.

De acordo com os dados apresentados, os estados mais eficientes em todos os períodos foram SP, RS, AM, RO e AP. Estes definem a fronteira e são referência para medir a eficiência dos demais. Note que há uma concentração de estados da região Norte. Os estados menos eficientes encontram-se localizados no Nordeste. Os estados com eficiência abaixo de 0,5, em ordem crescente são MA, AL, BA, PB, PA, PI, RN, CE e SE. Todos estes estados poderiam dobrar o nível de bem-estar utilizando a mesma quantidade de insumos. No período estudado, os estados que tiveram perda de eficiência foram $\mathrm{RO}$ e BA. Obtiveram ganhos de eficiência os estados de ES, GO, AC, CE, MT, PE, PI, SC, RJPR, RS, PB, MG, RN, AP, MS, MA, AL e SE, em ordem decrescente. Os demais estados mantiveram os seus níveis de eficiência.

Tabela 5

Eficiência técnica dos estados brasileiros

\begin{tabular}{ccccc}
\hline & \multicolumn{4}{c}{ Médias } \\
& $1986-1989$ & $1990-1992$ & $1993-1995$ & $1996-1998$ \\
\hline RO & 0,9250 & 0,7160 & 0,6570 & 0,7630 \\
AC & 0,6570 & 0,5780 & 0,8020 & 0,8500 \\
AM & 1,0000 & 1,0000 & 1,0000 & 1,0000 \\
RR & 1,0000 & 1,0000 & 1,0000 & 1,0000 \\
PA & 0,3790 & 0,4450 & 0,4610 & 0,3770 \\
AP & 0,9210 & 1,0000 & 1,0000 & 1,0000 \\
MA & 0,1620 & 0,1950 & 0,1810 & 0,2190 \\
PI & 0,2170 & 0,2580 & 0,3320 & 0,3840 \\
CE & 0,2350 & 0,3260 & 0,3650 & 0,4270 \\
RN & 0,3110 & 0,3380 & 0,3820 & 0,4030 \\
PB & 0,2410 & 0,3040 & 0,3260 & 0,3620 \\
PE & 0,3660 & 0,4360 & 0,4490 & 0,5320 \\
AL & 0,3110 & 0,3360 & 0,3010 & 0,3260 \\
SE & 0,4890 & 0,5090 & 0,4740 & 0,4980 \\
BA & 0,4090 & 0,4070 & 0,3860 & 0,3180 \\
MG & 0,5590 & 0,5790 & 0,6190 & 0,6670 \\
ES & 0,5610 & 0,7280 & 0,8190 & 0,9050 \\
RJ & 0,8070 & 0,9560 & 0,9230 & 0,9650 \\
SP & 1,0000 & 1,0000 & 1,0000 & 1,0000 \\
PR & 0,6660 & 0,7930 & 0,7250 & 0,8190 \\
SC & 0,8340 & 0,9350 & 0,9380 & 0,9950 \\
RS & 0,8600 & 1,0000 & 1,0000 & 1,0000 \\
MT & 0,5700 & 0,6010 & 0,6330 & 0,7490 \\
MS & 0,5420 & 0,6280 & 0,6490 & 0,5950 \\
GO & 0,3020 & 0,4670 & 0,5020 & 0,5130 \\
\hline Fonte: & Restlados & 010000 &
\end{tabular}

Fonte: Resultados calculados pelos autores.

Para possibilitar a visualização dos resultados, os valores da tabela 5 são representados na forma de gráfico na figura 2. 
Figura 2

Eficiência técnica dos estados brasileiros na geração de bem-estar - modelo 1

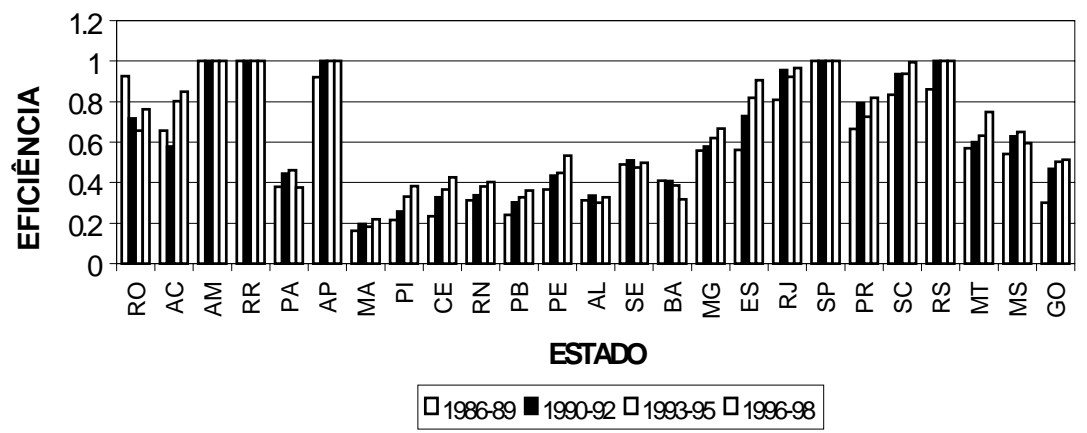

\subsection{Modelo 2 - Eficiência usando o PIB per capita como output}

Os resultados obtidos usando o PIB per capita como output são mostrados na Tabela 6. Comparando estes resultados com os obtidos pelo modelo 1, pode-se observar que os estados mais eficientes são os mesmos e que as medidas de eficiência técnica dos demais estados diferem pouco nos dois modelos estimados.

Entretanto, os resultados da eficiência técnica dos estados AC, PA, MA, PI, CE, RN, PB, AL, SE , BA, MG, ES, PR, MT, MS e GO são maiores no modelo 2. Isto significa que, em se tratando de produtividade em bens e serviços, estes estados estão mais próximos dos líderes. Este resultado é uma evidência de que os estados mais produtivos também são relativamente menos desiguais.

Por outro lado, os ganhos de eficiência foram maior no modelo 1 para os estados AC, PI, CE, RN, PE, MG, ES, RJ, PR, SC, RS, MT e GO. Isto significa que nestes estados a desconcentração da renda teve um papel importante na elevação da eficiência. 
Tabela 6

Eficiência técnica dos estados brasileiros

\begin{tabular}{|c|c|c|c|c|}
\hline \multicolumn{5}{|c|}{ Médias } \\
\hline & 1986-1989 & 1990-1992 & 1993-1995 & 1996-1998 \\
\hline $\mathrm{RO}$ & 0,8850 & 0,6790 & 0,6060 & 0,7090 \\
\hline $\mathrm{AC}$ & 0,7350 & 0,6000 & 0,8490 & 0,9060 \\
\hline $\mathrm{AM}$ & 1,0000 & 1,0000 & 1,0000 & 1,0000 \\
\hline $\mathrm{RR}$ & 1,0000 & 1,0000 & 1,0000 & 1,0000 \\
\hline PA & 0,4390 & 0,4700 & 0,4900 & 0,3980 \\
\hline $\mathrm{AP}$ & 1,0000 & 1,0000 & 1,0000 & 1,0000 \\
\hline MA & 0,1720 & 0,1980 & 0,1980 & 0,2460 \\
\hline PI & 0,2570 & 0,2820 & 0,3320 & 0,3940 \\
\hline $\mathrm{CE}$ & 0,2930 & 0,3660 & 0,4080 & 0,4800 \\
\hline $\mathrm{RN}$ & 0,3600 & 0,3880 & 0,4130 & 0,4490 \\
\hline PB & 0,2830 & 0,3480 & 0,3800 & 0,4300 \\
\hline $\mathrm{PE}$ & 0,4220 & 0,4790 & 0,4750 & 0,5650 \\
\hline $\mathrm{AL}$ & 0,3290 & 0,3510 & 0,3360 & 0,3680 \\
\hline $\mathrm{SE}$ & 0,5600 & 0,5470 & 0,5420 & 0,5730 \\
\hline BA & 0,4800 & 0,4550 & 0,4070 & 0,3240 \\
\hline MG & 0,6450 & 0,6360 & 0,6460 & 0,6930 \\
\hline $\mathrm{ES}$ & 0,6920 & 0,7830 & 0,8650 & 0,9620 \\
\hline RJ & 0,9370 & 1,0000 & 0,9280 & 0,9860 \\
\hline SP & 1,0000 & 1,0000 & 1,0000 & 1,0000 \\
\hline PR & 0,7380 & 0,8050 & 0,7490 & 0,8480 \\
\hline $\mathrm{SC}$ & 0,8720 & 0,9150 & 0,8740 & 0,9450 \\
\hline $\mathrm{RS}$ & 0,9410 & 1,0000 & 1,0000 & 1,0000 \\
\hline MT & 0,6510 & 0,6470 & 0,6670 & 0,8110 \\
\hline MS & 0,6010 & 0,5960 & 0,6570 & 0,6600 \\
\hline $\mathrm{GO}$ & 0,3630 & 0,5220 & 0,5400 & 0,5570 \\
\hline
\end{tabular}

Fonte: Resultados calculados pelos autores.

Comparando ainda os resultados deste modelo com os dos dois primeiros, observa-se que as médias de eficiência são maiores no modelo 2 , em todos os períodos selecionados. Isto demonstra que os estado são mais eficientes em gerar renda do que bem-estar. Além disto, a eficiência média cresce entre 1986 e 1998. Este resultado confirma a redução na dispersão da renda entre estados. A ineficiência na geração de renda no Brasil não é um fenômeno relacionado à distribuição da produtividade entre as regiões. Ela é resultado da ineficiência em distribuir renda entre os indivíduos dentro de cada estado.

\subsection{Modelo 3 - Eficiência usando o IDH como output}

Os resultados obtidos para o modelo com o Índice de Desenvolvimento Humano (IDH) como output, para os anos de 1986, 1995, 1996 e 1998, são mostrados na tabela 7. Neste caso, observa-se que os estados mais eficientes na geração de bemestar são: RO, AP, PI, PR e RS em 1986; RR, AP, ES, RS e MG, em 1995; RR, RS e MG em 1996 e RR, ES e RS, em 1998. 
Tabela 7

Eficiência técnica dos estados brasileiros

\begin{tabular}{|c|c|c|c|c|}
\hline \multicolumn{5}{|c|}{ Médias } \\
\hline & 1986 & 1995 & 1996 & 1998 \\
\hline $\mathrm{RO}$ & 1,0000 & 0,9190 & 0,9530 & 0,9610 \\
\hline $\mathrm{AC}$ & 0,9830 & 0,9610 & 0,8790 & 0,9500 \\
\hline $\mathrm{AM}$ & 0,9160 & 0,8590 & 0,8750 & 0,9030 \\
\hline $\mathrm{RR}$ & 0,9140 & 1,0000 & 1,0000 & 1,0000 \\
\hline PA & 0,9420 & 0,8370 & 0,8140 & 0,7930 \\
\hline $\mathrm{AP}$ & 1,0000 & 1,0000 & 0,9830 & 0,9570 \\
\hline MA & 0,9670 & 0,7120 & 0,7400 & 0,8070 \\
\hline PI & 1,0000 & 0,8250 & 0,7850 & 0,7890 \\
\hline $\mathrm{CE}$ & 0,8740 & 0,7730 & 0,7870 & 0,8050 \\
\hline $\mathrm{RN}$ & 0,9530 & 0,8600 & 0,8310 & 0,8210 \\
\hline PB & 0,9080 & 0,7760 & 0,7880 & 0,8080 \\
\hline $\mathrm{PE}$ & 0,8700 & 0,7490 & 0,7670 & 0,7780 \\
\hline $\mathrm{AL}$ & 0,7870 & 0,6540 & 0,6350 & 0,6700 \\
\hline $\mathrm{SE}$ & 0,9480 & 0,9760 & 0,9380 & 0,9670 \\
\hline $\mathrm{BA}$ & 0,8950 & 0,8000 & 0,8250 & 0,8190 \\
\hline MG & 0,9620 & 0,9390 & 0,9780 & 0,9590 \\
\hline $\mathrm{ES}$ & 0,9970 & 1,0000 & 0,9980 & 1,0000 \\
\hline RJ & 0,9870 & 0,9640 & 0,9890 & 0,9450 \\
\hline $\mathrm{SP}$ & 0,8980 & 0,9620 & 0,9690 & 0,9440 \\
\hline PR & 1,0000 & 0,9700 & 0,9990 & 0,9670 \\
\hline $\mathrm{SC}$ & 0,9690 & 0,9810 & 0,9360 & 0,9870 \\
\hline $\mathrm{RS}$ & 1,0000 & 1,0000 & 1,0000 & 1,0000 \\
\hline MT & 0,9820 & 1,0000 & 1,0000 & 0,9920 \\
\hline MS & 0,9770 & 0,9040 & 0,8920 & 0,9260 \\
\hline $\mathrm{GO}$ & 0,9820 & 0,8820 & 0,8930 & 0,8900 \\
\hline
\end{tabular}

Fonte: Resultados calculados pelos autores.

Comparando o ordenamento da eficiência dos estados no modelo 1 com o ordenamento no modelo 3, no ano de 1998, observa-se que ocorrem 21 alterações. Os estados que mais perderam posições no ordenamento foram AM, SP, PE, AP e RJ. Os estados que mais ganharam posição foram MT, SE, ES, BA e MA. Em termos de regiões, usando como critério a média das posições dos estados, o ordenamento sofre apenas uma modificação. No modelo 3 o Centro-Oeste ganha a terceira posição que pertencia ao Norte no modelo 1. Nas demais posições estão as regiões Sul, Sudeste e Nordeste, respectivamente, em primeiro, segundo e quinto lugares.

Os estados que tiveram variação positiva da eficiência foram RR, SP, SE, SC e MT. Este resultado mostra que a variação no IDH não acompanhou a variação na capacidade produtiva na maioria dos estados e, por isso, estes sofreram perdas de eficiência.

Pode-se notar que em nenhum dos anos selecionados o estado de São Paulo aparece com eficiência técnica igual a um, diferentemente dos valores dos modelos 
analisados anteriormente. A diferença entre os estoques per capita de capital e trabalho do estado de São Paulo (e da região Sudeste de modo geral) e dos estados da região Norte é muito maior que a diferença entre os IDH's. Neste sentido, seria provável que se os estados da região Norte possuíssem os mesmos níveis de capital e trabalho de São Paulo, os seus IDH's seriam maiores que deste último, mantidos os mesmos níveis de eficiência técnica.

As diferenças entre as medidas de eficiência entre os anos estudados podem ser melhor visualizadas no gráfico no figura 3 a seguir.

\section{Figura 3}

Eficiência técnica dos estados brasileiros na geração de bem-estar - modelo 3

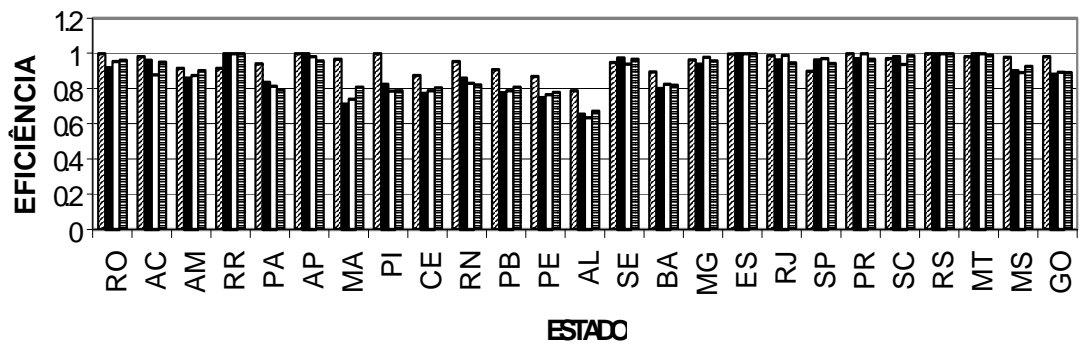

⿴囗十 1986 - 1995 口 1996 目 1998

O fato de estados pobres aparecerem entre os mais eficientes na geração de bem-estar pode ser melhor explicado visualizando-se a figura 4. Nele são grafados conjuntamente o IDH e os estoques de capital e de trabalho como proporção do IDH e dos estoques de capital e de trabalho do estado de São Paulo, respectivamente, tomando-se como exemplo o ano de 1991. Pode-se observar que a diferença dos estoques relativos de capital e trabalho entre os estados é bastante elevada. Por exemplo, no estado do Amapá o estoque de capital corresponde a 0,042 vezes o estoque de capital de São Paulo e o estoque de trabalho corresponde a 0,025. Entretanto, o IDH deste mesmo estado corresponde a 0,89 vezes o IDH de São Paulo. O IDH relativo do Amapá é 21,19 vezes maior do que o seu estoque de capital relativo e 35,6 vezes o estoque de trabalho relativo. Desta forma, justifica-se a maior eficiência do Amapá na geração de bem-estar, bem como de outros estados economicamente menos expressivos, em comparação a alguns estados mais ricos quando o IDH é utilizado como medida de bem-estar. Estes dados sugerem que a medida de bem-estar considerada não é proporcional ao nível de riqueza de uma região. Em outras palavras, não é apenas a riqueza que define o IDH. Em estados mais pobres é comum haver a produção para consumo próprio e outras formas de 
renda não monetárias. Além disso, nos estados mais pobres as transferências de recursos da união e os programas especiais de saúde e educação podem promover um crescimento do IDH proporcionalmente maior que o crescimento da capacidade produtiva.

Figura 4

Capital, trabalho e IDH dos estados brasileiros relativos aos valores de São Paulo no ano de 1991

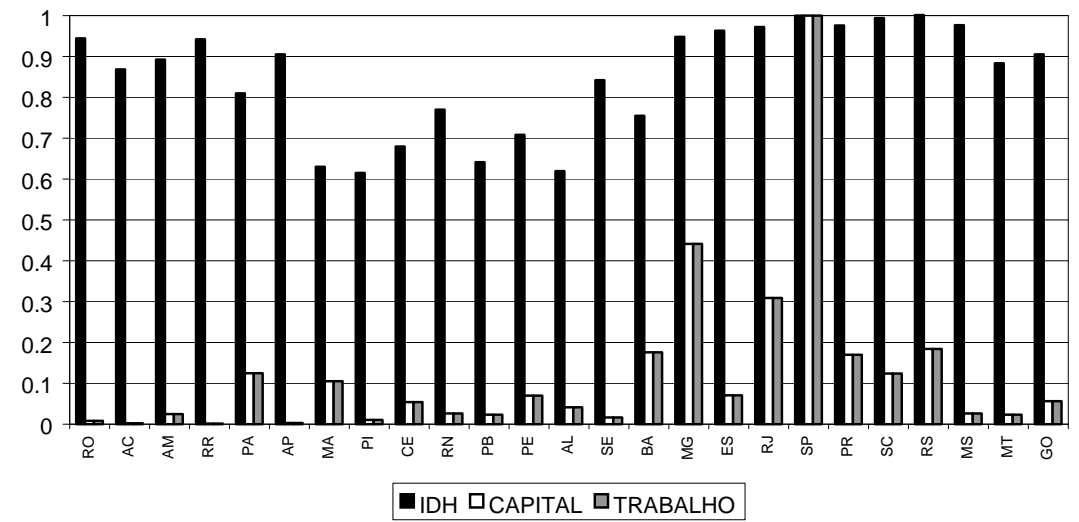

\section{Conclusão}

A análise da trajetória de desigualdade na distribuição de renda entre os estados brasileiros, de 1986 a 1998, mostrou que a desigualdade total sofreu uma redução significativa no período. Em relação a desigualdade entre estados de uma mesma região, verificou-se que, apenas nas regiões Nordeste e Sudeste, a desigualdade diminuiu significativamente.

No que se refere às medidas de eficiência na geração de bem-estar, os resultados mostram que em média as medidas de eficiência são maiores quando o output é o IDH. Este resultado se deve ao fato que a renda tem peso menor nesta medida de bem-estar. No entanto, deve ser observado que este resultado não classifica os estados brasileiros como detentores de IDH's dentro dos padrões dos países desenvolvidos. Usando a metodologia do DEA, sempre existirão estados considerados plenamente eficientes. Isto nem sempre corresponde a um estado com um IDH elevado. Quando a dispersão dos valores dos outputs é pequena, é possível que a eficiência média seja elevada mesmo quando os valores dos outputs sejam baixos, 
desde que os níveis dos inputs não sejam muito distintos.

Por sua vez, a média da eficiência é maior no modelo 2 que no modelo 1. A eficiência em gerar renda média é maior do que em gerar bem-estar social quando este é mensurado pela medida de Sen. Isto mostra que a eficiência produtiva é maior e cresce mais rápido do que a eficiência distributiva.

Quanto ao ordenamento dos estados por critério de eficiência, no modelo 2, os resultados são semelhantes aqueles obtidos pelo modelo 1. No entanto, quando se utiliza o modelo 3, alguns dos estados mais pobres, tais como os estados da região Norte, apresentam-se relativamente mais eficientes quando comparados com os estados mais ricos. Isto porque o diferencial na dotação de fatores de produção entre os mais ricos e os mais pobres é muito maior que o diferencial de IDH. Entretanto, existem estados que além de serem pobres, apresentam medidas de eficiência inferiores a alguns estados ricos. Entre os estados pobres destacam-se os da região Nordeste, que além de apresentarem uma baixa dotação de fatores de produção, são ineficientes na utilização dos mesmos.

\section{Referências}

Barros, R. P., Henriques, R., \& Mendonça, R. (2001). A estabilidade inaceitável: Desigualdade e pobreza no Brasil. IPEA. Texto para Discussão No. 800, Rio de Janeiro.

Barros, R. P. d., Mendonça, R. S. P., \& Duarte, R. P. N. (1997). Bem-estar, pobreza e desigualdade de renda: Uma avaliação da evolução histórica e das disparidades regionais. IPEA. Texto para Discussão No. 454.

Benevides, A. \& Ataliba, F. (2002). Os efeitos da abertura econômica sobre as desigualdades de renda pessoal nos estados brasileiros de 1986 a 1999 . VII Encontro Regional de Economia do Nordeste, 18 e 19 de Julho de 2002, Anais em CD.

Charnes, A., Cooper, W. W., \& Rhodes, R. (1978). Measuring efficiency of decision making units. European Journal of Operational Research, 2:429-444.

de Geografia e Estatística, I. I. B. (2000). Contas regionais - 1986-98. Extraído do site ftp://ftp.ibge.gov.br.

Deininger, K. \& Squire, L. (1998). New ways of looking at old issues. Journal of Development Economics, 57:259-87. 
Dollar, D. \& Aart, K. (2000). Growth is good for the poor. Policy Research Working Paper. World Bank, Washington DC.

Farrell, M. J. (1957). The measurement of productive efficiency. Journal of Royal Satatistical Society, Series A , CXX(3):253-290.

Fischer, R. D. (2001). The evolution of inequality after trade liberalization. Journal of Development Economics, 66:555-579.

IBGE - Instituto Brasileiro de Geografia e Estatística (2000a). Anuários estatísticos do brasil, anos de 1987 a 1997.

IBGE - Instituto Brasileiro de Geografia e Estatística (2000b). Pesquisa nacional por amostragem familiar - PNAD, anos de 1986 a 1998.

Kakwani, N. (1980). Income Inequality and Poverty: Methods of Estimation and Policy Applications. Oxford University Press, London.

Kuznets, S. (1955). Economic growth and income inequality. American Economic Review, 45(1):1-28.

Lambert, P. J. (1993). The Distribution and Redistribution of Income - A Mathematical Analysis. Manchester University Press, Manchester, 2nd edition.

Lipton, M. \& Ravallion, M. (1995). Poverty and policy. In Berhman, J. \& Srinivasan, T., editors, Handbook of Development Economics. North Holand, Amsterdam.

Lovell, C. A. K. (1993). Production frontier and productive efficiency. In Fried, H. \& Lovell, C. A. K. \& Schimidt, S. S., editors, The Measure of Productive Efficiency: Techniques and Applications, pages 3-67. Oxford University Press, New York.

Malmquist, S. (1953). Index number and indiference curves. Trabajos de Estatistica, 4(1):209-42.

Marinho, E., Ataliba, F., \& Lima, F. (2001). Produtividade, variação tecnológica e variação da eficiência das regiões e estados brasileiros. ANPEC. XXIX Encontro Nacional de Economia, Salvador.

Marinho, E. \& Benegas, M. (2002). Avaliação Inter/Intra-regional de produtividade total dos estados e regiões brasileiras: Uma abordagem não-paramétrica utilizando o conceito de meta-fronteira de produção. Latin American Meeting of the Econometric Society. 24 a 27 de julho de 2002. Anais em CD. 
Mukhopadhaya, P. (2001). Efficiency criteria and the sen-type social welfare function. National University of Singapore. Departament of Economics, Working Paper No. 0114.

Ravallion, M. \& Chen, S. (1997). What can new survey data tell us about recent changes in distribution and poverty. World Bank Economic Review, 11(2).

Sen, A. K. (1973). On Economic Inequality. Clarendon Press, Oxford.

Theil, H. (1989). The development of international inequality, 1960-85. Journal of Econometrics, 42:145-155.

Theil, H. (1996). Studies in Global Econometrics. Kluwer Academic Publishers, Boston.

Thorbecke, E. \& Hong-Sang, J. (1996). A multiplier decomposition method to analyze poverty alleviation. Journal of Development Economics, 48(2):279-300. 